Dom. Cien., ISSN: 2477-8818

Vol. 4, núm.4., oct, 2018, pp. 284-307

\title{
Las Finanzas Publicas en el Ecuador y su incidencia en la economía del país
}

\section{Public Finance in Ecuador and its impact on the country's economy}

\section{Finanças Públicas no Equador e seu impacto na economia do país}

\author{
Walter M. Mendoza-Zamora ${ }^{\mathrm{I}}$ \\ walter.mendoza@unesum.edu.ec \\ Carmen M. García-Álava II \\ marcelagarciaalava@gmail.com
}

\author{
Jessenia H. Morán-Chilán III \\ jessenia.moran@unesum.edu.ec \\ Gabriela E. Muñiz-Salazar IV \\ gabyxb_2802@hotmail.com
}

Recibido: 07 de septiembre de 2018 * Corregido: 28 de septiembre de 2018 * Aceptado: 25 de octubre de 2018

I. Gestor Financiero, Ingeniero Comercial, Master en Gestión Empresarial, Docente de la Universidad Estatal del Sur de Manabí, Jipijapa, Ecuador.

II. Ingeniera en Contabilidad y Auditoría, Magister en Contabilidad y Finanzas, Docente de la Universidad Laica Eloy Alfaro de Manabí, Manta, Ecuador.

III. Economista, Master en Economía Agraria, Coordinadora del Departamento de Cultura de la UNESUM, Docente de la Universidad Estatal del Sur de Manabí, Jipijapa, Ecuador.

IV. Doctora en Medicina, Profesional, Hospital Básico de Jipijapa, Ecuador. 


\section{Resumen}

En la actualidad, los hogares ecuatorianos afrontan la realidad que viven las finanzas de la economía familiar, de ahí el propósito de entender conceptos aparentemente complejos, como lo es el referido a la elaboración del presupuesto nacional, lo cual supone la estimación de los ingresos que serán percibidos de diferentes fuentes para posteriormente depositarlos en la cuenta Única Tesoro Nacional y, a los egresos, entendidos como los recursos que el estado consigna a las diferentes instituciones del sector público de manera permanente para que puedan ejecutar los programas, proyectos y obras que han sido aprobadas y se encuentran en la planificación institucional, asegurando de esa forma, la provisión continua de bienes y servicios sociales y la mejora en las condiciones de vida de las personas. Por ende, las finanzas públicas vienen a representar los diferentes elementos que forman parte de un estado y contribuyen al funcionamiento de un país, a través del manejo de los ingresos y los gastos, mejorando la calidad de vida de la población mediante el desarrollo sostenible y sustentable para futuras generaciones. De allí, que el presente apartado tiene como objetivo analizar las finanzas públicas del estado Ecuatoriano y su incidencia en la economía del país, teniendo en cuenta el estado como un ente encargado de observar las políticas económicas y asegurar la correcta colocación de rubros en el sistema presupuestal, a través del manejo de los ingresos y los gastos.

Palabras claves: Estado, Economía, Sector Publico, Finanzas Públicas, Sistema Presupuestal, Políticas Publicas. 


\begin{abstract}
Currently, Ecuadorian households face the reality of the finances of the family economy, hence the purpose of understanding apparently complex concepts, such as the one referred to the preparation of the national budget, which involves estimating the income that They will be perceived from different sources and then deposited in the Single National Treasury account, and to the expenditures, understood as the resources that the State consigned to the different institutions of the public sector permanently so that they can execute the programs, projects and works that have been they have been approved and are in the institutional planning, thus ensuring the continuous provision of social goods and services and the improvement in the living conditions of the people. Therefore, public finances come to represent the different elements that are part of a state and contribute to the functioning of a country, through the management of income and expenses, improving the quality of life of the population through sustainable development and sustainable for future generations. Hence, that this section aims to analyze the public finances of the Ecuadorian state and its impact on the economy of the country, taking into account the state as an entity in charge of observing economic policies and ensuring the correct placement of items in the system budget, through the management of income and expenses.
\end{abstract}

Key words: State, Economy, Public Sector, Public Finances, Budgetary System, Public Policies. 


\section{Introducción.}

El Estado, es una sociedad humana, asentada permanentemente sobre un determinado territorio, constituida y regida por un orden jurídico que es creado, establecido y aplicado por un poder soberano, para obtener el bien público temporal. Sus finanzas vienen a estar representadas por la actividad económica del sector oficial, las mismas comprenden los bienes, rentas y deudas que forman el activo y el pasivo de la Nación y todos los demás bienes $\mathrm{y}$ rentas cuya administración corresponde al Poder Nacional, a través de las distintas instituciones creadas por el estado para tal fin. Para Cett (2015):

El Estado, representa un organismo jurídico y político, el cual debe estar encaminado a garantizar fines sociales y el bienestar del pueblo: estableciéndose para ello, normativas y políticas vinculadas a la atención de las necesidades de su población para la vida plena del ser humano como individuo y como sujeto social.(p.76).

Lo descrito lleva a considerar el deber que tiene el estado de garantizar la estabilidad social para con su pueblo; el mismo, dentro de sus funciones debe respaldar los medios necesarios para la producción de finanzas públicas y servicios, en el cumplimiento de los fines, económicos o extraeconómicos, señalados por la clase política responsable del Gobierno de la Nación. En el mismo orden Paredes y Heath (2014), establecen:

La actividad financiera de toda Nación debe nutrirse de la planificación y el manejo eficiente de sus recursos; de allí, que debe existir una correcta administración, la cual gire sus ordenanzas mediante políticas sociales asertivas, que busquen alcanzar los objetivos de orden público, traduciéndose los mismos, en bienestar social, como la 
función fundamental del Estado, su complejidad, sus objetivos y la actividad para lograrlos. (p.75)

En virtud de esto, se puede entender que las políticas públicas deben estar enmarcadas en un contexto global dirigido al desarrollo de lineamientos que garanticen el progreso social; lo que implica un método estructural, histórico y totalizante basado en la igualdad de oportunidades sociales, políticas y económicas.

Desde esta perspectiva, se presentan las finanzas públicas, entendidas como aquellos recursos financieros manejados a través de políticas nacionales, para el funcionamiento y garantía de la estabilidad económica de cada Nación, estableciéndose con ello, el mejoramiento de la calidad de vida de sus habitantes. Ante, lo descrito, Paredes y Heath (ob cit), refieren:

Las finanzas públicas de cada Nación se encuentran dadas por las recaudaciones, precio de petróleo y endeudamiento. Las mismas, provienen del Presupuesto General del Estado, basándose en la estimación los recursos financieros que posee el país, con el fin de solventar el cumplimiento de proyectos de inversión. (p.76)

Dicho de otra forma, las finanzas públicas vienen a constituir la actividad económica del sector social, a través de una estructura peculiar que convive con la economía de mercado, es decir, las mismas forman parte de aquello que pertenece a todos los ciudadanos, incluyendo en ello, su inversión, prioridad de gasto y la planificación para el desarrollo.

En base a estos señalamientos, se establece la importancia de analizar las finanzas públicas en el Ecuador y su incidencia en la economía del país. 


\section{Método.}

La metodología implícita en el presente apartado es de tipo documental, dado que, cada una de las actividades a ejecutar partirán de la revisión de documentos, trabajos, reportes bibliográficos, los cuales serán tomados como fuente para la descripción de cada uno de los elementos integradores de las variables en curso. En tal sentido, Cáceres (2013), define a los estudios documentales "como actividades representadas en acciones de revisión bibliográfica que responden a una estructuración dada por el investigador "(p. 22).

De allí, que el mismo, interpreta la teoría analizada de manera descriptiva, lo que la hace caracterizar a los estudios documentales, por cuanto llevan al investigador a profundizar en la indagación, para luego exponer sus criterios de interpretación en función a los planteamientos establecidos en cada uno de los objetivos propuestos para su desarrollo.

\section{Técnicas de Recolección de los Datos}

Los estudios documentales debido a sus particularidades de un apoyo bibliográfico, conducen a la introducción de técnicas eminentemente viables; a fin de ofrecer una verdadera gama de alternativas encargadas de facilitar su interpretación. Al respecto, Terán (2011), indica las técnicas documentales “deben responder a diversas preguntas tales como: qué, cómo, porqué y para qué, sólo a través de la solución de estas interrogantes el investigador las adecua a sus necesidades" (p. 24). A razón de lo planteado, se hace necesario expresar que, en la presente investigación se utilizan técnicas que corresponden al trabajo intelectual, por lo que se recurre a la consulta de fuentes; entre las cuales se encuentran: la técnica del resumen y la técnica de fichaje. 
El Resumen: consiste en una exposición breve de las ideas principales de un contenido. Para Terán (ob cit) este método se basa en "la exposición condensada de un escrito en el cual se reflejan fielmente las ideas expresadas en el texto original" (p.113). He aquí, la importancia que tiene para un trabajo documental la aplicación del resumen, dado que el mismo facilita la comprensión de lo analizado mediante la selección minuciosa de aquello que se considera más relevante en un texto.

Las fichas de trabajo, las cuales en toda investigación documental cumplen con el objeto de registrar la información y conservarla para cuando se requiera de su utilidad. Por tal razón, la utilización de esta ficha permitió a al investigador seleccionar un contenido específico, el cual es extraído de los documentos y objetos de estudio, interpretados y estudiados.

Finalmente, se plantean las fichas bibliográficas o de identificación de las obras en las cuales se plasmaron datos tales como: títulos, editorial, lugar, fecha de edición entre otros. Así como también, las fichas textuales, donde se recogieron informaciones, tal y como aparece en el documento. Por último, se encuentran las fichas personales, que tienen como función seleccionar anotaciones, producto de la interpretación de los datos consultados.

\section{Resultados.}

\section{Estado Ecuatoriano}

La Constitución de la República define al Ecuador como un Estado social de derecho, soberano, unitario, democrático, pluricultural y multiétnico, que se rigen en normas y leyes en acciones públicas y privada enfocado en los derechos humanos, que establecen el bien común, de esta manera se maneja un estado que está regido bajo la constitución de la República del Ecuador que limita el poder político, teniendo como primicia el bienestar de los ciudadanos.

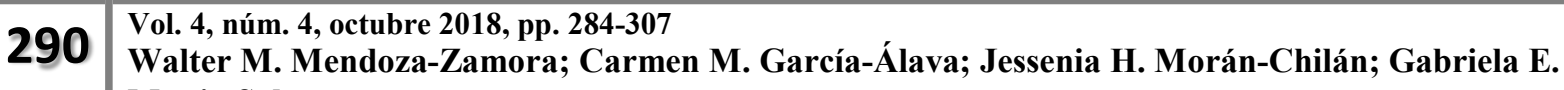


En base a este señalamiento, se puede definir al estado Ecuatoriano como el encargado de manejar sus recursos y en base a ello aplicar leyes que estén dirigidas a alcanzar el bienestar general de la Nación. Su poder emana de la unidad del país, y debe, entre sus funciones proteger el medio ambiente y defenderlo. Así también, debe establecer políticas públicas que logren eliminar la pobreza, y cuidar que sea la voluntad del pueblo la que guíe al país. Entre sus funciones se encuentra el poder legislativo, ejecutivo y judicial. (Anonimo, 2013).

El estado Ecuatoriano, a través de sus poderes debe hacer valer los derechos de las personas, buscando con ello que los mismos no sean vulnerados. Del mismo modo, debe garantizar el manejo eficiente de sus finanzas públicas, considerándose para ello, a través de sus instituciones debe encargarse de administrar los recursos del país, gestionando su distribución en el presupuesto general del estado, generando bienestar sostenible sobre la población.

\section{Sector Público}

Según (Arnoletto, 2007), "se entiende por sector público a un conjunto de actividades económicas de un país, que presenta características peculiares y homogéneas”. (p. 23). Entiéndase con ello que, el sector público es aquel que incluye las actividades que están dentro de la esfera gubernamental: la administración, las empresas nacionalizadas, el sistema impositivo, la banca oficial, la seguridad social, las corporaciones públicas.

Por consiguiente, el sector público está entendido como una parte del estado, quien se encarga de desempeñar diferentes funciones para ejecutar actividades en los sectores económicos primarios, secundarios y terciarios además se encarga de normar y regular dichas actividades para que exista un equilibrio en la economía; también proporciona servicios de seguridad y como ente benefactor

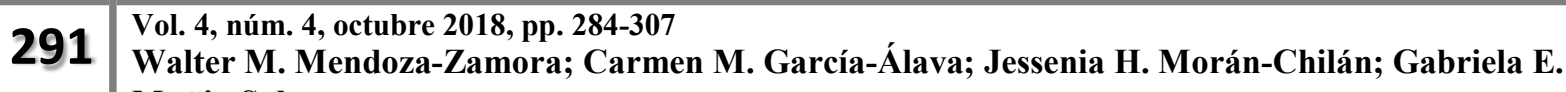


proporciona servicios de salud, beneficencia y educación gratuita; todo ello con la finalidad que las personas tengamos una mejor situación económica y poder mejorar la calidad de vida. Toda entidad u organizaciones que prestan bienes y servicios a la sociedad se las conoce como sector Público. Ante estas ideas, Lara (2014), señala:

El sector público comprende los organismos y dependencias de las funciones Ejecutiva, Legislativa, Judicial, Electoral y de Transparencia y Control Social. Está conformado por los ministerios públicos, Ministerio de Gobierno, Cultos, Policía y Municipalidades; Ministerio de Relaciones Exteriores, Ministerio de Defensa Nacional, Ministerio de Economía y Finanzas, Ministerio de Educación y Cultura, Ministerio de Obras Públicas y Comunicaciones, Ministerio de Trabajo y Empleo, Ministerio de Agricultura y Ganadería; gobiernos autónomos descentralizados, Prefecturas, municipios, juntas parroquiales, empresas públicas; la Banca pública, como el Banco Nacional del Ecuador; Corporación Financiera Nacional, las instituciones de Seguridad Social; entre otras. (p.44)

De igual forma, Lara (ob cit), menciona "que la administración Pública en principio es la organización encargada de ejecutar las políticas que son resueltas en los niveles representativos del poder político". (p.45). Su organización es jerárquica, con un flujo descendente de órdenes y un flujo ascendente de responsabilidad ante el superior.

\section{Competencia administrativa del estado}

Se entiende por competencia administrativa del estado a la encargada de velar por los recursos económicos generados por cada Nación. En otras palabras, es el conjunto de atribuciones de los

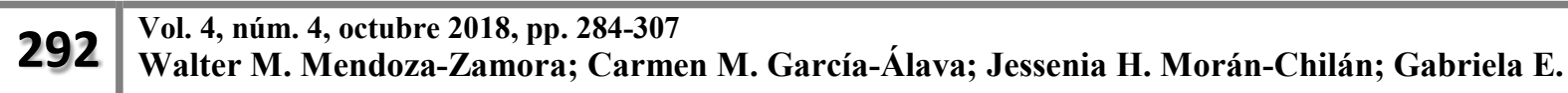


órganos y entes que componen el Estado, las mismas que son precisadas por el ordenamiento jurídico. La importancia de la competencia es tal, que sin ella el acto administrativo deviene en nulo. (Napurí, 2008)

Cada organismo del sector público debe desempeñarse dentro de los límites y especificaciones señaladas de las competencias atribuidas según su función y debe hacer una contribución especial, haciendo lo mejor para servir a la ciudadanía en general, prestando atención de calidad y respondiendo a las necesidades de la población y por los recursos asignados.

\section{Economía}

El sistema económico es social y solidario, es aquel que reconoce al ser humano como sujeto y fin; el mismo, propende a una relación dinámica y equilibrada entre sociedad, estado y mercado, en armonía con la naturaleza; y tiene por objetivo garantizar la producción y reproducción de las condiciones materiales e inmateriales que posibiliten el buen vivir.

En el caso de las finanzas públicas, la economía actuará de manera integrada, a través de las formas de organización económica pública, privada, mixta, popular y solidaria, y las demás que la Constitución determine. En todo caso, la economía popular y solidaria se regulará de acuerdo con la ley e incluirá a los sectores cooperativistas, asociativos y comunitarios. (Ecuador, Constitucion del Ecuador , 2010)

De allí, que el desarrollo de la economía ecuatoriana y del manejo de la política fiscal, que es el problema central de este estudio, se observa, durante el período de análisis se va observando una deuda pública a gran escala, según indicadores del FMI se observa una participación de cooperación, pero una baja en el generador de economía del ecuador que es el petróleo.

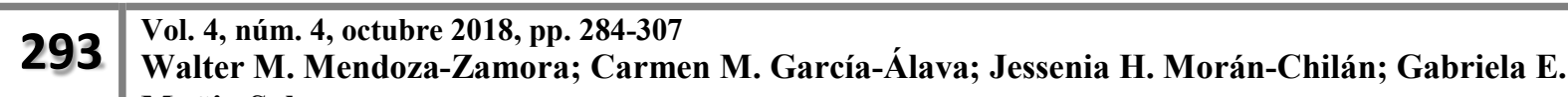


Sistema económico y política económica

Según el Art. 283.- El sistema económico es social y solidario; reconoce al ser humano como sujeto y fin; propende a una relación dinámica y equilibrada entre sociedad, Estado y mercado, en armonía con la naturaleza; y tiene por objetivo garantizar la producción y reproducción de las condiciones materiales e inmateriales que posibiliten el buen vivir. (Ecuador, Constitucion del Ecuador , 2010)

Se entiende a través de esta normativa que, las políticas económicas direccionan sus objetivos para conseguir una economía sostenible a través de sus ingresos y su riqueza nacional, garantizando la participación de empresa y microempresa en el mercando nacional a su vez impulsado la matriz productiva como futuro generador de economía del país. El sistema económico se integrará por las formas de organización económica, según lo que Constitución determine. La economía popular y solidaria se regulará de acuerdo con la ley e incluirá a los sectores cooperativistas, asociativos y comunitarios.

Asimismo, se encuentra el artículo 284 que estipula el ministerio de finanzas en las normas técnicas, los objetivos principales es la adecuada distribución de la riqueza nacional, incentivando el crecimiento económico como primicia la matriz productiva, de esta manera asegura soberanía alimentaria y energética, a su vez promociona el desarrollo equilibrado nacional entre el campo y la ciudad convirtiendo el socio cultura en una base llena de valores y estabilidad.

Art. 284.- La política económica tendrá los siguientes objetivos:

1. Asegurar una adecuada distribución del ingreso y de la riqueza nacional. 
2. Incentivar la producción nacional, la productividad y competitividad sistémica, la acumulación del conocimiento científico y tecnológico, la inserción estratégica en la economía mundial y las actividades productivas complementarias en la integración regional.

3. Asegurar la soberanía alimentaria y energética.

4. Promocionar la incorporación del valor agregado con máxima eficiencia, dentro de los límites biofísicos de la naturaleza y el respeto a la vida y a las culturas.

5. Lograr un desarrollo equilibrado del territorio nacional, la integración entre regiones, en el campo, entre el campo y la ciudad, en lo económico, social y cultural.

6. Impulsar el pleno empleo y valorar todas las formas de trabajo, con respeto a los derechos laborales.

7. Mantener la estabilidad económica, entendida como el máximo nivel de producción y empleo sostenibles en el tiempo.

8. Propiciar el intercambio justo y complementario de bienes y servicios en mercados transparentes y eficientes.

9. Impulsar un consumo social y ambientalmente responsable ${ }^{1}$. (Ecuador, Ministerio de Finanzas, 2010)

\section{Política económica en ecuador}

Las políticas económicas representan las herramientas que usa el estado para monitorear el capital nacional y alcanzar objetivos propios, estas herramientas fundamentan el crecimiento y sostenibilidad económico y a su vez interviene directamente con el empleo y la estabilidad en los

\footnotetext{
${ }^{1}$ Su objetivo es Mantener la estabilidad económica desarrollando programas que generen ingresos direccionados en la matriz productiva.

Archivo recuperado de la página; http://www.finanzas.gob.ec/normas-tecnicas-del-sinfip/
}

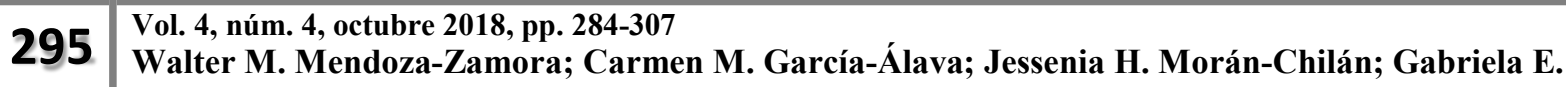


precios, son basados según el PIB y sus indicadores, de esta manera intervine el estado con políticas para saber administrar recursos tangibles e intangibles en periodos según sus mandatos.

Según lo señalado, la magnitud y complejidad del problema que enfrenta la economía ecuatoriana no pueden ser ·evaluados a partir, únicamente, de indicadores de la profunda recesión de los últimos tres años, ya que ha existido en el país una tendencia creciente hacia la separación entre la economía moderna comercial y la economía tradicional. (Lefeber, 2015).

El problema del desarrollo económico en Ecuador, bien sea en una economía de mercado basada en la empresa privada, o bien dentro de una organización política y económica socialista, exige un aumento generalizado de la productividad del trabajo, ya que sus políticas públicas no pueden guiarse con los mandatos ejercidos por otras Naciones, específicamente lo que es el caso de países vecinos como es cuba, Venezuela.

Por consiguiente, es importante resaltar que las finanzas del Gobierno central en Ecuador reflejan que Esta Nación destaca dentro del grupo por su incremento en la recaudación tributaria y se mantiene alineado a la tendencia regional de aumentar la participación de los impuestos directos en su estructura tributaria. De igual forma, se destaca entre el grupo de Naciones, como el país que más ha incrementado el gasto público en los rubros de capital y compra de bienes y servicios. Finalmente, resalta su estructura de gastos inflexible a la baja, lo cual vulnera la sostenibilidad de rubros estratégicos para lograr un mayor desarrollo humano, como la infraestructura y la inversión social. 


\section{Política fiscal}

Las políticas fiscales que orientan las finanzas del ecuatoriano buscan el crecimiento socioeconómico junto con la inversión extranjera a su vez impulsando la matriz productiva, considerando para ello, las instituciones públicas permitida, las cuales, podrán financiarse bajo tasas de contribuciones independientemente del presupuesto que el genera el estado ecuatoriano, esta se generar mediando a la obtención de recurso financieros bajo el pago de impuesto y otros ingresos como es la venta de petróleo

Art. 285.- La política fiscal tendrá como objetivos específicos:

1. El financiamiento de servicios, inversión y bienes públicos.

2. La redistribución del ingreso por medio de transferencias, tributos y subsidios adecuados.

3. La generación de incentivos para la inversión en los diferentes sectores de la economía y para la producción de bienes y servicios, socialmente deseables y ambientalmente aceptables.

Art. 287.- Toda norma que cree una obligación financiada con recursos públicos establecerá la fuente de financiamiento correspondiente.

Solamente las instituciones de derecho público podrán financiarse con tasas y contribuciones especiales establecidas por ley. 


\section{Presupuesto General del Estado:}

Es el instrumento para la determinación y gestión de los ingresos y egresos del Estado, e incluye todos los ingresos y egresos del sector público, con excepción de los pertenecientes a la seguridad social, la banca pública, las empresas públicas y los gobiernos autónomos descentralizados.

Programación Institucional:

Comprende la especificación de objetivos, políticas y metas de corto, mediano y largo plazo acorde con los planes estratégicos y plurianuales institucionales y con los planes nacionales, sobre cuya base se elaboran los planes operativos a través de la correspondiente programación anual, en la que se definen las actividades y proyectos con sus respectivas metas e indicadores de gestión. La responsabilidad recae en las unidades de planificación y programación institucional.

\section{Programación Presupuestaria:}

Es la fase del proceso presupuestario en la que, sobre la base de los planes de desarrollo y las políticas gubernamentales, sectoriales e institucionales, se definen las propuestas de gestión de corto plazo, expresándolas en actividades y proyectos que busquen cumplir con los objetivos de la planificación.

\section{Presupuesto:}

El presupuesto constituye una previsión de ingresos, financiamiento, gastos y amortizaciones. También se lo define como el instrumento por medio del cual se determinan y proyectan las fuentes de recursos, que permitirán financiar las autorizaciones máximas de gastos, para un período anual,

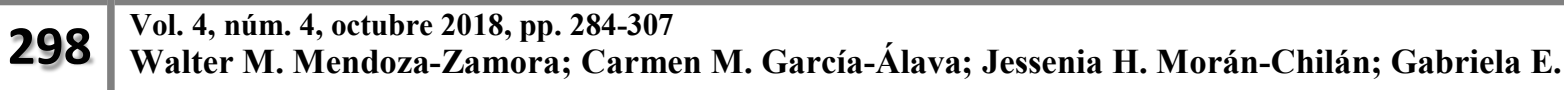


con el propósito de ejecutar los programas de gobierno y alcanzar sus objetivos y metas, todo ello orientado a satisfacer las necesidades básicas de la población contribuyendo de esta manera al buen vivir El presupuesto a través de la asignación de recursos vincula los programas y proyectos con la planificación. El presupuesto puede tener el nivel de institucional, regional, local o nacional.

\section{Finanzas}

Finanzas es el sitio de la economía que estudia el funcionamiento de los mercados de dinero y capitales, en ellos operan las políticas de captación de recursos, el valor del dinero en el tiempo y el coste del capital. Las mismas, estudian cómo los agentes económicos (empresas, familias o Estado) deben tomar decisiones de inversión, ahorro y gasto en condiciones de incertidumbre. Al momento de tomar estas decisiones los agentes pueden optar por diversos tipos de recursos financieros tales como: dinero, bonos, acciones o derivados, incluyendo la compra de bienes de capital como maquinarias, edificios y otras infraestructuras. Ver diferencia entre ahorro e inversión. (Madison, 2016)

\section{Economía actual}

El financiamiento de un país radica de tres factores muy importantes donde se encierra la parte más recaudadora del en donde se generan recursos para solventar gastos, estos se identifican de acuerdo en estos seis últimos años.

En primer lugar, la recaudación tributaria, una recaudación más eficiente, la recaudación tributaria pasó de 14\% del PIB en 2011 a 19\% del PIB en 2017. (Macón, 2017)

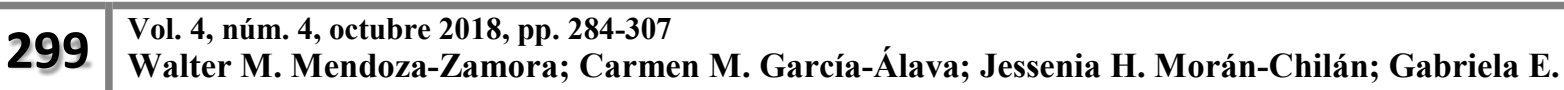


Una segunda fuente de financiamiento fueron los altos precios del petróleo, que permitieron contar con crecientes ingresos.

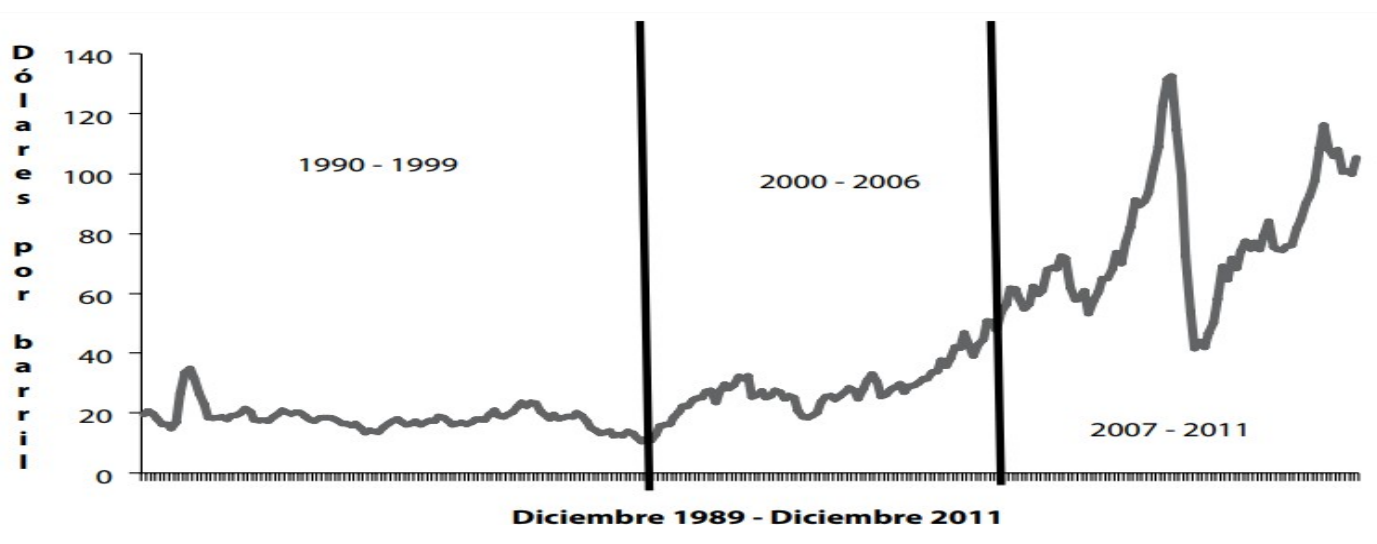

Ilustración 1.- Precio promedio mensual petróleo crudo WTI.

Fuente: http://www.indexmundi.com/es/precios-de-mercado/?mercancia=petroleo-crudo-texas\&meses $=300$

La tercera fuente de financiamiento es el endeudamiento, interno y externo. En el ámbito interno, el principal financista del Gobierno fue el IESS y en cuanto a deuda externa, China, la CAF y el BID. (Meneses, 2014)

De acuerdo a datos estadísticos los saldos de deudas publica tanta interna como externa creció un 40\% del año 2012 ha 2018 y estimación crecerá 8 puntos del PIB en el 2019, esto se debe a gastos en educación, vivienda, salud, entre otros gastos que estipula el estado a beneficio de la ciudadanía. Finalmente, resalta su estructura de gastos inflexible a la baja, lo cual vulnera la sostenibilidad de rubros estratégicos para lograr un mayor desarrollo humano, como la infraestructura y la inversión social 
El saldo total de deuda pública (interna y externa) creció 40\% entre 2016 y 2018, incluyendo el efecto de la recompra de los bonos Global 12 y 30. Este crecimiento se concentra entre 2018 y 2019, ya que en estos años la deuda pública total creció 48\%, lo que indica una mayor dependencia de fuentes externas para financiar el gasto público, esto aumentaría 8 puntos del PIB. Adicionalmente, existe un cambio en la estructura del 18 endeudamiento, con concentración de deuda en operaciones de plazos cortos y altos costos. (Anda, 2016)

De igual forma, Ecuador se destaca dentro del grupo de países que más ha incrementado el gasto público en los rubros de capital y compra de bienes y servicios.

Según la Secretaria Nacional de Planificación y Desarrollo, 2012. Folleto Informativo 1: Transformación de la Matriz productiva indica:

La economía ecuatoriana se ha caracterizado por ser proveedora de materias primas en el mercado internacional y al mismo tiempo importadora de bienes y servicios de mayor valor agregado. Los constantes e imprevistos cambios en los precios internacionales de las materias primas, así como su creciente diferencia frente a los precios de los productos de mayor valor agregado y alta tecnología, han colocado a la economía ecuatoriana en una situación de intercambio desigual sujeta a los vaivenes del mercado mundial.

Consciente de esta situación, se inició el proceso de cambio del patrón de especialización productiva de la economía que le permita al Ecuador generar mayor valor agregado a su producción en el marco de la construcción de una sociedad del conocimiento. Por ello, lograr transformar la matriz productiva es uno de los desafíos del país, el que permite al Ecuador superar el modelo de

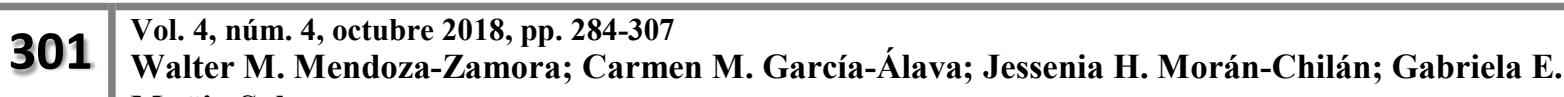


generación de riquezas: concentrador, excluyente y basado en recursos naturales, por un modelo democrático, incluyente y fundamentado en el conocimiento y las capacidades de las y los ecuatorianos.

\section{Afectación de la economía}

Desde fines del año pasado, ocho años de administración de la bonanza parecían sucumbir precipitadamente ante la obligada gestión de la austeridad, por la mala administración de recursos el estado ecuatoriano se vio involucrado en una crisis demasiada alta en datos porcentuales con disminución en el precio del petróleo, una recesión económica por el gatos público en los rubros de capital esto se dio, por inversiones no sujetas a un estudio técnico con un margen de error demasiado alto que se vio implicada el estado que disminuyó su deuda externa apenas un $0,1 \%$.

En el mandado actual se implementaron estrategias para la recaudación de recursos emanados de la recaudación tributaria, el cual representa uno de los factores más eficientes de la economía Ecuatoriana, y el más representativo a la hora de enfrentar la crisis económica generadas por la política del anterior mandatario. En tal sentido, es necesario expresar que las finanzas públicas en Ecuador se encuentran limitadas, tanto por la desaceleración de la economía como por la caída del precio del petróleo. Los países que registran un mayor índice de PIB per cápita son Estados Unidos, Alemania y Francia, mientras que Ecuador se ubica como el país con el menor PIB per cápita de la región. (Ekos, 2017)

\section{Conclusiones.}

Las finanzas públicas incluyen el manejo de conceptos y diferentes medios de actuación que contribuyen a lograr los objetivos que tiene el estado mediante la buena administración y la

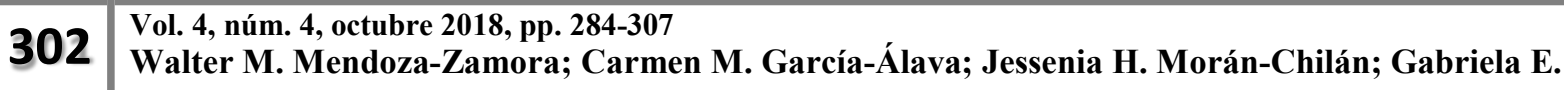


asignación apropiada de recursos lo que incide de manera radical en el ejercicio de las competencias de los funcionarios y/o servidores del sector público quienes deben actuar de manera honesta, teniendo en consideración que para que se logren las planificaciones propuestas y en ejecución se necesita la integración y compromiso de todos los actores directos e indirectos con la finalidad común de beneficiar y mejorar la calidad de vida de la población.

Tal y como se señala anteriormente, las finanzas públicas representan el escenario nacional de la gestión desarrollada por la administración de un período mandatario. De aquí, que para la fecha actual, reportes periodísticos e informativos de Gestión Digital, sostienen que la proforma de finanzas en Ecuador 2019 contempla un crecimiento de 1,43\% que, si bien es bajo, aún es optimista. De hecho, en CORDES se considera que el crecimiento económico del próximo año será menor que el de 2018 (estimado en 1,1\% - 1,2\% según las últimas declaraciones de la gerente del Banco Central). Tres motivos principales llevan a pensar que las finanzas del sector público de esta Nación contemplarán:

- Una previsible ralentización en la entrega de créditos por parte de los bancos, es decir, (en 2018 la tasa de crecimiento de la cartera de créditos ha superado por mucho a la de los depósitos);

- Habrá un también previsible fortalecimiento del dólar como consecuencia del aumento de las tasas de la Reserva Federal de EE.UU. (lo que afectará a la ya golpeada competitividad del sector productivo nacional, el cual no permite proyectar un repunte en las exportaciones no petroleras medidas en volumen)

- Se propiciará el propio ajuste en el gasto que proyecta el Gobierno y que puede ser mayor al esperado si escasean las fuentes de financiamiento. 
En ese contexto, es de vital importancia señalar que si el crecimiento económico en Ecuador para el año 2019 es menor al contemplado en la proforma, es esperable que los ingresos tributarios también sean más bajos. En cuanto a los ingresos petroleros, la proforma se basa en un aumento en la producción de crudo (que, de hecho, explicaría en buena parte el crecimiento previsto para la economía en su conjunto) y en un precio de referencia de $\$ 58,3$ por barril, muy superior al precio de \$ 41,9 contemplado en el PGE de 2018.

Finalmente, el Gobierno actual expresa que espera recibir en 2019 \$ 1.000 millones por concepto de "concesiones". No obstante, ante esta noticia hay que agregar que, no se cuenta con la información suficiente para hacer un análisis detallado, pero en principio, tomando en cuenta el antecedente de presupuestos de años anteriores (en los que se incluyó ingresos por posibles ventas de activos estatales que nunca se concretaron), el Gobierno podría haberse mostrado más cauto en ese rubro.

En efecto, a través del escenario de ingresos y gastos descrito, el Gobierno actual Ecuatoriano espera que sus finanzas en 2019 lleguen a un déficit global de \$ 3.655 millones y a un déficit primario (sin considerar el pago de intereses) de \$ 290 millones. Incluso con este escenario, que podría deteriorarse si el físco no recibe los ingresos estimados en la proforma, las necesidades de financiamiento serán de \$ 8.166 millones, es decir, \$ 300 millones menos que lo estimado en el Presupuesto codificado para 2018.

En ese contexto de posible estrechez financiera, es deseable que el Gobierno Ecuatoriano se acerque al FMI para avanzar en un programa de financiamiento de mediano plazo, el cual le permita cubrir una parte importante de la brecha de 2019 y, a la vez, lleve al Ejecutivo a ser más decidido en

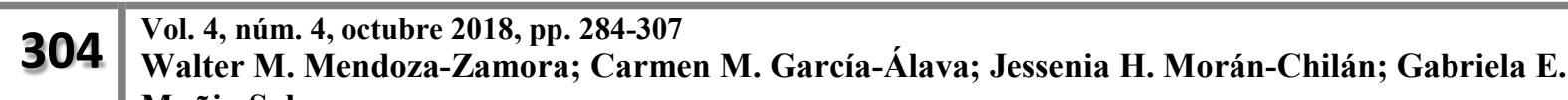


algunas acciones en las que hasta ahora se muestra demasiado tímido (recorte del gasto, revisión de subsidios, reforma laboral, etc.).

En conclusión, más allá de las críticas a una proforma y gestión pública actual, que muestra recortes insuficientes en el gasto y, a primera vista, un excesivo optimismo en los ingresos, es necesario que el esfuerzo para ordenar las cuentas públicas no recaiga únicamente sobre el Gobierno Central (que es el que está cubierto en el PGE), sino que se extienda también al resto del sector público, principalmente gobiernos seccionales y empresas públicas.

\section{Bibliografía.}

Anda, D. (2016). Crecimiento, finanzas públicas. Quito: Udla. Obtenido de https://www.udla.edu.ec/cie/wp-content/uploads/2016/09/CrecimientoFinanzas-DanielaAnda-Karla-Meneses.pdf

Angulo, M. O. (s.f.). EL COMERCIO. Obtenido de http://www.elcomercio.com/actualidad/leninmoreno-acciones-presupuesto-economiaecuador.html

Anonimo. (2018 de 08 de 2013). Ecotec. Obtenido de http://www.ecotec.edu.ec/documentacion\%5Cinvestigaciones\%5Cestudiantes\%5Ctrabajos_d e_clases/30800_pasantia_251.doc

Arnoletto, E. J. (2007). Glosario de Conceptos Políticos Usuales. Obtenido de Ed. EUMEDNET : http://www.eumed.net/dices/listado.php?dic=3

Comercio, E. (s.f.). El comercio. Obtenido de Terremoto del 16 de abril: https://www.metroecuador.com.ec/ec/noticias/2015/12/30/2015-ano-de-las-protestas-contrael-gobierno.html

Ecuador, C. d. (19 de 09 de 2010). Constitucion del Ecuador . Obtenido de http://repositorio.dpe.gob.ec/bitstream/39000/638/1/NN-001-Constituci\%C3\%B3n.pdf

Ecuador, C. d. (19 de 09 de 2010). Ministerio de Finanzas. Obtenido de http://www.ecotec.edu.ec/documentacion\%5Cinvestigaciones\%5Cestudiantes\%5Ctrabajos_d e_clases/30800_pasantia_251.doc 
ECUADOR, M. D. (03 de DICIEMBRE de 2010). MANUAL DE PROCEDIMIENTOS DEL SISTEMA DE PRESUPUESTO. Obtenido de $\mathrm{http}$ //diccionario.administracionpublica.gob.ec/adjuntos/manuel-de-procedimientos.pdf

ECUADOR, M. D. (SEPTIEMBRE de 2012). CODIGO ORGANICO DE PLANIFICACION Y FINANZAS PUBLICAS. Obtenido de http://www.finanzas.gob.ec/wpcontent/uploads/downloads/2012/09/CODIGO_PLANIFICACION_FINAZAS.pdf

Ekos. (30 de Marzo de 2017). Ekosnegocios. Obtenido de Economía Ecuatoriana una comparación internacional:

http://www.ekosnegocios.com/negocios/verArticuloContenido.aspx?idArt=5559

EL TELEGRAFO. (08 de 04 de 2017). Obtenido de https://www.eltelegrafo.com.ec/noticias/economia/8/ecuador-supera-la-recesion-economicay-cerrara-el-2017-con-un-crecimiento-del-1-42

EUMED. (2015). Obtenido de http://www.eumed.net/cursecon/ecolat/ec/2015/matrizproductiva.html

FAO, G. (15 de Diciembre de 2010). www.fao.org. Obtenido de www.fao.org: http://www.fao.org/tempref/docrep/fao/006/w9633s/W9633S00.pdf

Finanzas, M. d. (s.f.). NORMATIVA DEL SISTEMA DE ADMINISTRACION FINANCIERA. Obtenido de http:/www.finanzas.gob.ec/wpcontent/uploads/downloads/2013/08/Normativa_Presupuesto_Contabilidad_Tesorer\%C3\%A Da_Acuerdo_447.pdf

\section{Lefeber, L. (2015). LA ECONOMIA POLITICA. Quito: CORPORACION EDITORA NACIONAL .}

Macón, J. (2017). Economía del sector público. Mc Graw Hill, capítulos 1 y 3.

Madison, R. (28 de 03 de 2016). Economipedia. Obtenido de http://economipedia.com/definiciones/finanzas.html

Mayorga, Á. C. (27 de 04 de 2012). El Estado y su importancia. Obtenido de El telegrafo: https://www.eltelegrafo.com.ec/noticias/cartas-al-director/1/el-estado-y-su-importancia

Meneses, K. (28 de Agosto de 2014). Indexmundi. Obtenido de http://www.indexmundi.com/es/precios-de-mercado/?mercancia=petroleo-crudotexas\&meses $=300$

Muñoz, R. R. (10 de 2017). Observatorio Economía Latinoamericana. ISSN: 1696-8352. Obtenido de EUMED: http://www.eumed.net/cursecon/ecolat/ec/2017/realidad-socioeconomicaecuador.html

Napurí, C. G. (20 de 02 de 2008). LA COMPETENCIA. Obtenido de http://blog.pucp.edu.pe/blog/christianguzmannapuri/2008/02/20/la-competencia/ 
Nivel socieconomico. (s.f.). Obtenido de http://definicionyque.es/nivel-socioeconomico/

Perspectiva 2015. (s.f.). Obtenido de https://cosas.com.ec/perspectivas-del-2015-\%C2\%93elsuceso-que-me-marco\%C2\%94/

PLAN NACIONAL PARA EL BUEN VIVIR. (2013). Obtenido de https://www.google.com.ec/url?sa=t\&source=web\&rct=j\&url=http://www.unicef.org/ecuado r/Plan_Nacional_Buen_Vivir_20132017.pdf\&vd=2ahUKEwj46cCWodbZAhUGH5AKHbWfDrUQFjADegQIAxAB\&usg=AO vVaw1H9gX46iO2w_5MnUFoK5cn

SENPLADES. (2012). Obtenido de http://www.planificacion.gob.ec/wpcontent/uploads/downloads/2013/01/matriz_productiva_WEBtodo.pdf 